\title{
Mutación FTL3-ITD y su relación con variables hematológicas y clínicas en individuos con Leucemia Mieloide Aguda
}

\author{
FTL3-ITD mutation and its relationship with hematological and clinical variables in \\ individuals with Acute Myeloid Leukemia
}

\section{A mutação FTL3-ITD e sua relação com variáveis hematológicas e clínicas em indivíduos com leucemia mielóide aguda}

\author{
Fanny Calderón Calle \\ fvikicalderon@hotmail.com \\ ORCID: 0000-0003-2076-9776
}

\author{
Carem Francelys Prieto Fuenmayor \\ carem.prieto@ucacue.edu.ec \\ ORCID: 0000-0002-7752-932X
}

Universidad Católica de Cuenca, Cuenca-Ecuador

Recibido 20 de noviembre 2020 | Arbitrado y aceptado 9 de diciembre 2020 | Publicado en 04 de enero 2021

\begin{abstract}
RESUMEN
Introducción. La Leucemia Mieloide Aguda es la neoplasia hematológica más común, caracterizada por una proliferación incontrolada de células madre hematopoyéticas. La mutación FLT3/ITD se presenta en aproximadamente el $30 \%$ de todos los pacientes con ésta patología, se asocia con mayor riesgo de recaída y menor supervivencia. El FLT3-ITD puede usarse como un factor pronóstico de la gravedad de ésta patología, importante para predecir los resultados clínicos en pacientes con LMA. Objetivo. El objetivo de este estudio fue relacionar la mutación FLT3/ITD con variables hematológicas y clínicas en pacientes diagnosticados con Leucemia Mieloide Aguda atendidos en la Sociedad de Lucha contra el Cáncer (SOLCA) de la ciudad de Cuenca, periodo 2013 -2020. Métodos. Se obtuvieron los datos a partir de registros secundarios registrados una base de datos del hospital, el universo de la muestra lo constituyeron 63 pacientes, diagnosticados con LMA, se les analizó la mutación FLT3/ITD por PCR Convencional. Resultados. Se encontró la presencia de la mutación en un 9.5\% y una asociación significativamente estadística con alteraciones hematológicas relacionados con niveles de hemoglobina anormal $(p=0,037)$ y ratio 6,63 y LDH elevada en 1,21 veces $(p=0,024)$; recuento elevado de leucocitos y blastos $(p=0,031)$. Los individuos portadores de la mutación se presentó con mayor incidencia en el sexo masculino y grupo etario adulto mediano (45-64 años). Conclusiones. La literatura internacional afirma que la mutación FLT3/ITD en un importante marcador pronóstico; debido a su baja frecuencia, no se pudo determinar una relación estadísticamente significativa con otras variables clínicas en este estudio.
\end{abstract}

Palabras clave: Duplicación Interna en Tándem; Leucemia Mieloide Aguda; Lactato Deshidrogenasa; Tirosina quinasa

\begin{abstract}
Introduction. Acute Myeloid Leukemia is the most common hematological neoplasm, characterized by an uncontrolled proliferation of hematopoietic stem cells. The FLT3 / ITD mutation occurs in approximately $30 \%$ of all patients with this pathology, it is associated with a higher risk of relapse and lower survival. FLT3-ITD can be used as a poor prognostic factor, important for predicting clinical outcomes in patients with AML. Objective. The objective of this study was to characterize the FLT3 / ITD mutation and its relationship with hematological and clinical variables in patients diagnosed with Acute Myeloid Leukemia treated at SOLCA in the city of Cuenca, period 2013-2020. Methods. Data were obtained from secondary records in a hospital database, the universe of the sample was made up of 63 patients, diagnosed with AML, and the FLT3 / ITD mutation was analyzed by Conventional PCR. Results. The presence of the mutation was found in $9.5 \%$ and a statistically significantly association with hematological alterations related to abnormal hemoglobin levels $(p=0.037)$ and ratio 6.63 and LDH elevated in 1.21 times $(p=$ $0.024)$; Elevated leukocyte and blast count $(p=0.031)$. Individuals carrying the mutation had a higher incidence in males and in the middle adult age group (45-64 years). Conclusions. The international literature affirms that the FLT3 / ITD mutation is an important prognostic marker; Due to its low frequency, it was not possible to determine a statistically significant relationship with other clinical variables in our study, for which it is suggested to expand the unirverse of the sample.
\end{abstract}

Key words: Internal Tandem Duplication; Leukemia Myeloid Acute; Lactate dehydrogenase; Tyrosine kinase

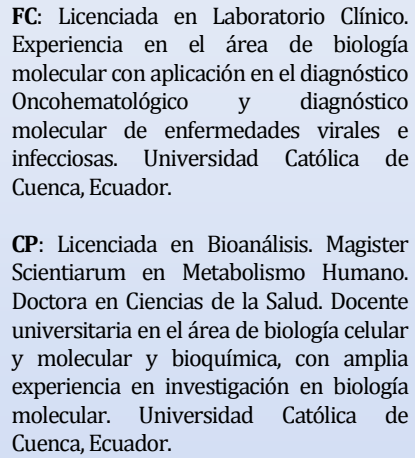

FC: Licenciada en Laboratorio Clínico. Experiencia en el área de biología molecular con aplicación en el diagnóstico Oncohematológico y diagnóstico molecular de enfermedades virales e infecciosas. Universidad Católica de Cuenca, Ecuador.

CP: Licenciada en Bioanálisis. Magister Scientiarum en Metabolismo Humano. Doctora en Ciencias de la Salud. Docente universitaria en el área de biología celular y molecular y bioquímica, con amplia experiencia en investigación en biología molecular. Universidad Católica de Cuenca, Ecuador. 
FC: Licenciada en Laboratorio Clínico. Experiencia en el área de biología molecular con aplicación en el diagnóstico Oncohematológico y diagnóstico molecular de enfermedades virales e infecciosas. Universidad Católica de Cuenca, Ecuador.

CP: Licenciada en Bioanálisis. Magister Scientiarum en Metabolismo Humano. Doctora en Ciencias de la Salud. Docente universitaria en el área de biología celular y molecular y bioquímica, con amplia experiencia en investigación en biología molecular. Universidad Católica de Cuenca, Ecuador.

\begin{abstract}
RESUMO
Introdução. A Leucemia Mielóide Aguda é a malignidade hematológica mais comum, caracterizada pela proliferação descontrolada de células-tronco hematopoiéticas. A mutação FLT3/ITD está presente em aproximadamente $30 \%$ de todos os pacientes com esta patologia, e está associada a um maior risco de recaída e menor sobrevida. 0 FLT3-ITD pode ser usado como um fator prognóstico para a gravidade desta patologia, importante para prever os resultados clínicos em pacientes com LMA. Objetivo. 0 objetivo deste estudo foi relacionar a mutação FLT3/ITD com variáveis hematológicas e clínicas em pacientes diagnosticados com leucemia mielóide aguda tratados na Sociedade de Luta contra o Câncer (SOLCA) na cidade de Cuenca, período 2013 202020. Métodos. Os dados foram obtidos de registros secundários registrados em um banco de dados hospitalar, o universo da amostra consistiu de 63 pacientes diagnosticados com AML, eles foram analisados para a mutação FLT3/ITD por PCR convencional. Resultados. A presença da mutação foi encontrada em 9,5\% e uma associação estatística significativa com alterações hematológicas relacionadas a níveis anormais de hemoglobina $(p=0,037)$ e relação 6,63 e LDH elevada em 1,21 vezes $(\mathrm{p}=0,024)$; contagem elevada de leucócitos e explosões $(\mathrm{p}=0,031)$. Os indivíduos portadores da mutação ocorreram com maior incidência no sexo masculino e na faixa etária média adulta (45-64 anos). Conclusões. A literatura internacional afirma que a mutação FLT3/ITD em um marcador prognóstico importante; devido a sua baixa freqüência, uma relação estatisticamente significativa com outras variáveis clínicas não pôde ser determinada neste estudo.
\end{abstract}

Palavras-chave: Duplicação Tandem interna; Leucemia mielóide aguda; Desidrogenase láctica; Tirosina quinase

\section{INTRODUCCIÓN}

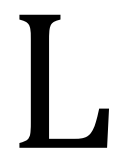
as Leucemias Mieloides Agudas (LMA) son un grupo de trastornos hematológicos malignos de progresión rápida. Representan entre el 15 y el $20 \%$ de las leucemias agudas en niños y el $80 \%$ en adultos (1). Les cataloga como un conjunto de enfermedades fenotípica y genéticamente heterogéneas, originadas por la acumulación de mutaciones en una célula madre hematopoyética; la duplicación interna en tándem en el gen de la tirosina quinasa 3 similar a FMS (FLT3/ITD), es una de las mutaciones de gran importancia para su estudio (2).

FLT3 pertenece a la familia de receptores tirosina quinasa (TK) de clase III;es un receptor de membrana, con un dominio TK, que regula la diferenciación y la proliferación celular (3), está localizado en el cromosoma 13q12, y comprende 24 exones que varían en su tamaño desde 83 pb hasta 562 pb, y abarcan $100 \mathrm{~kb}$ aproximadamente (1). Las mutaciones en el gen FLT3 son las más frecuentes en LMA $y$ dentro de ellas las duplicaciones internas en tándem (FLT3-ITD) en el dominio yuxtamembarana (4). Las mutaciones FLT3 / ITD se agrupan en exónes (11 al 15) del gen FLT3. Estas mutaciones normalmente consisten en una secuencia insertada en el marco análogo al fragmento de la proteína entre los aminoácidos de la proteína FLT3 $(5,6)$. Esta alteración de la secuencia nucleotídica se relaciona con mayores tasas de recaída y disminución de supervivencia, con una prevalencia entre $20 \%$ y $27 \%$. FLT3/ITD es especialmente frecuente en pacientes con cariotipo normal y con anormalidad cromosómica de la t $(15,17,(4,7,8)$.

En pacientes con LMA, se asocia con un alto porcentaje de células blásticas, mayor riesgo de recaída por remisión completa y supervivencia menor $(9,10)$. Estudios de correlación detallan que la mutación FLT3/ITD se observa en pacientes masculinos preponderantemente con recuentos de 
leucocitos altos y lactato deshidrogenasa (LDH) elevados. También se correlaciona en pacientes con FAB M1 y M4 $(11,12)$.

Debido a la importancia que tiene el diagnóstico de la mutación FLT3/ITD en pacientes con LMA es importante obtener este dato ya que los pacientes que presentan la mutación tienen un pronóstico desfavorable, con una mayor tasa de recaída y una supervivencia general inferior en comparación con los pacientes con LMA sin esta mutación (13).

Actualmente en Ecuador, no existen estudios que relacionen la incidencia del gen FLT3 con LMA. El diagnóstico oportuno, permitirá un manejo adecuado de pacientes con LMAya que este tipo de leucemias, tienen un comportamiento agresivo que puede provocar la muerte a corto plazo. Un tratamiento acertado mejora la sobrevida del paciente, permitiéndole a su vez una mejor calidad de vida.

El presente estudio tuvo como objetivo relacionar la mutación FLT3/ITD con variables hematológicas y clínicas en pacientes diagnosticados con Leucemia Mieloide Aguda atendidos en la Sociedad de Lucha contra el Cáncer (SOLCA) de la ciudad de Cuenca en Ecuador, periodo $2013-2020$

\section{MATERIALES Y MÉTODOS}

$\mathrm{E}$ l universo del estudio estuvo conformado por los registros de los pacientes diagnosticados con LMA en SOLCA en la ciudad de Cuenca en Ecuador, durante el periodo 2013-2020, de los cuales para la muestra del estudio se usaron un total de 63 registros pacientes aislados los cuales constituyen la totalidad de la muestra. Para este estudio se incluyeron los individuos que fueron diagnosticados con LMA e individuos a los que se realizó la detección molecular FLT3/ITD y se excluyeron los individuos que fueron diagnosticados con LMA pero que tuvieron una concentración de ARN menor de $10 \mathrm{ng} / \mu$ y un radio de pureza menor a 2 .

En cuanto a los métodos, técnicas e instrumentos de investigación o recolección de datos, los datos sociodemográficos y de interés médico clínico fueron obtenidos a partir de registros secundarios en una base de datos del hospital, donde se tuvo acceso previa aprobación del Comité Científico de la institución.

En relación a la extracción del ARN se realizó a partir de sangre periférica o médula ósea utilizando el protocolo indicado con el reactivo de TRIzol LS Reagent (ambión), con modificaciones del Laboratorio de Biología Molecular SOLCACuenca.

En un tubo de centrifuga de $1.5 \mathrm{ml}$ se agregó $1 \mathrm{ml}$ de TRIzol, se añadió $200 \mu$ de sangre periférica o médula ósea, aplicando vortex por $15 \mathrm{sg}$, añadiendo $200 \mu$ de cloroformo, se aplicó vortex por $15 \mathrm{sg}$, centrifungando a $10000 \mathrm{rev}$ por $10 \mathrm{~min}$ a $4^{\circ} \mathrm{C}$.

En un tubo de centrifuga de $1.5 \mathrm{ml}$ se adicionó 600ul de isopropanol y se agregó el sobrenadante del tubo centrifugado, se mezcló por inversión, llevando a centrifugación a $10000 \mathrm{rev}$ por $10 \mathrm{~min}$ a $4^{\circ} \mathrm{C}$ y desechando el sobrenádate del tubo.

Se Agregó $1 \mathrm{ml}$ de ethanol al 70\% al botón de precipitado, aplicando vortex suavemente, llevando a centrifugación a $10.000 \mathrm{rev}$ por $10 \mathrm{~min}$ a $4^{\circ} \mathrm{C}$, se desechó el sobrenadante, se incubó $10 \mathrm{~min}$ a $37^{\circ} \mathrm{C}$ para secar, se agregaron $50 \mu$ de agua grado molecular para rehidratar el botón de precipitado, incubando la muestra a 
$56^{\circ} \mathrm{C}$ por 5 min y guardando la muestra en un congelador a $-30^{\circ} \mathrm{C}$ para su conservación.

La técnica de cuantificación del ARN total se realizó mediante espectrofotometría a $260-280 \mathrm{~nm}$, para esto se utilizó un espectrofotómetro GeneQuant pro, en el que se introdujeron $12 \mu$ de cada una de las muestras, determinando la concentración en ng/ $\mu \mathrm{y}$ el radio de pureza de cada una de las muestras. Las muestras reunieron los requisitos de pureza y concentración.

Para la amplificación del cDNA el volumen total de la reacción en cadena de la Polimerasa (PCR) para cada una de las muestras se utilizó, agua grado molecular $1 \mu$, Random Primer $2 \mu$, (Invitrogen) y ARN $7.5 \mu$, y se llevó al termociclador 10 $\min$ a $65 \mathrm{C}$.

Posteriormente se preparó una mezcla para la PCR en donde se utilizó, buffer $5 \mathrm{x} 4$ $\mu$ (Invitrogen), DTT 0.1 M $2 \mu$ (Invitrogen) y DNTP's 10 mM (Invitrogen) $2 \mu$, RNAout $0.5 \mu$ (Invitrogen). Se agregó a cada tubo $8.5 \mu$ de la mezcla, y $1 \mu$ de Superscrit III (Invitrogen). Cada uno de los tubos de reacción contenía $12.5 \mu$ de la mezcla y 7.5 $\mu$ de muestra de ARN.

Cada reacción se llevó a cabo en un termociclador modelo 9700 de Applied Byosistems, el programa de termociclado empezó con una hibridación a $65^{\circ} \mathrm{C}$ por 10 min, seguido por el protocolo de amplificación 1 ciclo de $60 \mathrm{~min}$ a $37 \mathrm{C}, 1$ ciclo de $5 \mathrm{~min}$ a $99 \mathrm{C} \mathrm{y} 5^{\circ} \mathrm{C}$ por $5 \mathrm{~min}$.

Para la amplificación del Gen FLT3/ITD por PCR punto final se utilizó, agua grado molecular $35 \mu$ (Invitrogen), Buffer $10 \quad x \quad$ Tipo II $5 \mu$ (Applied Biosystems), MgCl2 25 Mm $10.5 \mu$ (Applied Biosystems), DNTPS $10 \mathrm{Mm} 3 \mu$ (Applied Biosystems), PRIMER FW $3 \mu$ (Invitrogen), PRIMER RW $3 \mu$ (Invitrogen), AmpliTaq Gold 250U (5U/ $\mu) \quad 0.5 \quad \mu$ (Appied Biosystems). Cada uno de los tubos contenía un volumen final $47 \mu$ de la mezcla más $3 \mu$ del cDNA.

La secuencia de los cebadores utilizados para la amplificación de FLT3/ITD fue:

\section{FLT3 FW 5' CAA GTA CAA AAA GCA ATT TAG G 3 '} FLT3 RW 5' GCT GTC TGC TTT TTC TTT CAG 3'

Cada reacción se llevó a cabo en un termociclador modelo 9700 Applied Biosystems, el programa de termociclado empezó con una incubación a $94{ }^{\circ} \mathrm{C}$ por 10 min, seguido por 40 ciclos de $30 \mathrm{~s}$ a $94{ }^{\circ} \mathrm{C}$, $30 \mathrm{~s}$ a $55,5^{\circ} \mathrm{C}$ y 1 min a $72{ }^{\circ} \mathrm{C}$. Además, un ciclo final de extensión a $72{ }^{\circ} \mathrm{C}$ por $5 \mathrm{~min}$.

La electroforesis se realizó en gel de agarosa al $2 \%$, al cual se adicionó $10 \mu$ de bromuro de etidio. $\mathrm{Al}$ producto amplificado se le agregó $3 \mu$ de azul de bromofenol. Se realizó una corrida constante de $120 \mathrm{~V}$ por $45 \mathrm{~min}$. Los resultados fueron visualizados utilizando un transiluminador UV, utilizando un equipo de foto documentación, observándose la banda amplificada del control interno a 265 pb y una banda a 360 pb correspondiente al FLT3 mutado.(Ver Figura 1). 


\section{5}

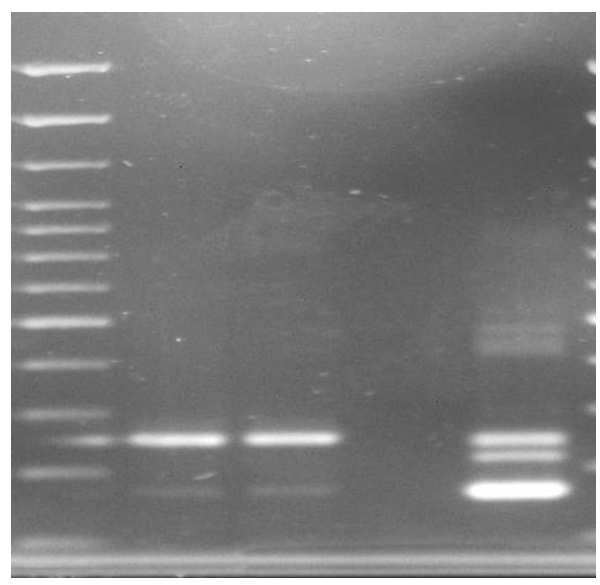

\begin{tabular}{|l|}
\hline FLT3/ITD \\
\hline $265 \mathrm{pb} \mathrm{Cl}$ \\
\hline
\end{tabular}

Figura 1. Gel de agarosa, donde se observa los productos de amplificación por RT-PCR para la mutación FLT3/ITD en 2 pacientes (carriles 2,3); control negativo (carril 4); control positivo FLT3/ITD (carril 5). La banda inferior corresponde al gen normal y la banda superior al gen FLT3 mutado. Las muestras fueron corridas en un gel de agarosa al $2 \%$ teñido con bromuro de etidio; en el carril 1 se utilizó un marcador de peso molecular de 100pb.

Para el procesamiento, resumen y presentación de la información (Tablas, Gráficos y Técnicas de análisis estadístico) se generó una base de datos en el programa SPSS 15, las variables cualitativas se exponen en tablas de frecuencia y porcentajes, para conocer la normalidad de las variables se utilizó la prueba de Kolmogórov-Smirnov, se aplicó para las variables que resulten normales, se utilizaron medidas de tendencia central media para variables no normales la mediana y el rango intercuartil. Se realizaron las frecuencias alélicas $\mathrm{y}$ genotípicas de la mutación y para realizar contrastes entre grupos por variables se utilizó la prueba de U de Mann- Whitney. Para verificar la asociación entre la presencia de la mutación y la alteración de las variables de estudio se utilizó ODD ratio, intervalo de confianza de $95 \%$, un valor será estadísticamente significativo cuando la p. sea menor a 0.05 .

El procedimiento de investigación siguió las normas éticas para la investigación con seres humanos, establecidas en la declaración de Helsinki, específicamente en sus adendum Declaración de Taiwan del año 2016, Declaración de la Asociación Médica Mundial (AMM), sobre las consideraciones éticas de las bases de datos de salud y biobancos. Se contó con la aprobación de la Comisión Científica del Instituto del Cáncer de la ciudad de Cuenca para la utilización de los datos para la investigación. El uso de los datos derivará en beneficio total de la sociedad como fue planteado en la justificación de la investigación. Se protegerá la dignidad, autonomía, privacidad y confidencialidad de los participantes en la investigación en todo momento. Los datos serán resguardados siendo usados exclusivamente por el investigador para los fines declarados en esta investigación. Los resultados presentados serán totalmente anónimos y los datos estarán codificados, de modo que se asegure la privacidad del paciente. Los 
pacientes podrán retirar sus datos cuando lo deseen.

\section{RESULTADOS}

$\mathrm{L}$ a muestra total constituyo el registro de 63 pacientes diagnosticados con LMA, los cuales cumplieron con los criterios de inclusión establecidos para este estudio. De estos, 27 corresponden a pacientes de sexo femenino que representa el 42,9\%, 36 pacientes son de sexo masculino que corresponde a 57,1\%. En el grupo etario, el 9,7\% (6) corresponde a pacientes que se encentran en el rango de 0 a 9 años, 10 pacientes $(16,1 \%)$ se encontraban en el rango de edad entre 10 a 19 años, dentro del rango de edad catalogada como adulto joven (20 a 44 años) también otros 19 pacientes que corresponde al 30,6\%, el $24,2 \%$ (15) se encuentran dentro del rango de 45 a 64 años, por último encontramos 12 pacientes $(19,4 \%)$ mayores a 64 años. La mayoría de pacientes con la mutación FLT3/ITD fueron del sexo masculino (4/ 66,7\%). Dentro del grupo etario correspondiente a adulto mediano encontramos 3 pacientes $(50,0 \%), 2$ pacientes $\quad(33,3 \%)$ corresponden al grupo de adultos mayores (Ver Tabla 1).

Tabla 1. Variables sociodemográficas en pacientes diagnosticados con LMA evaluados según sexo, grupo etario y diagnóstico de la mutación FLT3/ITD atendidos en SOLCA de la ciudad de Cuenca periodo 2013-2019.

\begin{tabular}{|c|c|c|c|c|c|c|c|}
\hline \multirow{3}{*}{\multicolumn{2}{|c|}{$\begin{array}{l}\text { Variables } \\
\text { sociodemográficas }\end{array}$}} & \multicolumn{6}{|c|}{ MUTACIÓN FLT3/ITD } \\
\hline & & \multicolumn{2}{|c|}{ TODOS } & \multicolumn{2}{|c|}{ NEGATIVO } & \multicolumn{2}{|c|}{ POSITIVO } \\
\hline & & $\mathbf{n}$ & $\%$ & $\mathbf{n}$ & $\%$ & $\mathbf{n}$ & $\%$ \\
\hline \multirow{4}{*}{ SEXO } & Femenino & 27 & 42,9 & 25 & 43,9 & 2 & 33,3 \\
\hline & Masculino & 36 & 57,1 & 32 & 56,1 & 4 & 66,7 \\
\hline & Niñez 0-9 & 6 & 9,7 & 6 & 10,7 & 0 & 0,0 \\
\hline & $\begin{array}{l}\text { Adolescente 10- } \\
19\end{array}$ & 10 & 16,1 & 10 & 17,9 & 0 & 0,0 \\
\hline \multirow{3}{*}{$\begin{array}{l}\text { Grupo } \\
\text { etario }\end{array}$} & $\begin{array}{l}\text { Adulto joven } \\
20-44\end{array}$ & 19 & 30,6 & 18 & 32,1 & 1 & 16,7 \\
\hline & $\begin{array}{l}\text { Adulto mediano } \\
45-64\end{array}$ & 15 & 24,2 & 12 & 21,4 & 3 & 50,0 \\
\hline & $\begin{array}{l}\text { Adulto mayor } \\
>64\end{array}$ & 12 & 19,4 & 10 & 17,9 & 2 & 33,3 \\
\hline
\end{tabular}

Edad mediana (rango intercuarticulo): Todos= $35(21,5)$; Negativo=34(21,5); Positivo= $56(11,5)$.

La presencia de la mutación FLT3/ITD, se encontró en 6 pacientes positivos que corresponde al 9,5\%, 57 pacientes resultaron negativos (90,5\%). (Ver Tabla 2). 
Tabla 2. Presencia de la Mutación FLT3/ITD en pacientes con LMA

\begin{tabular}{lcc}
\hline Diagnóstico de la Mutación FLT3/ITD & n & \% \\
\hline Negativo & 57 & 90,5 \\
Positivo & 6 & 9,5 \\
Total & 63 & 100,0 \\
\hline
\end{tabular}

El subtipo morfológico más frecuente fue la LMA M0 con un 20,6\% (13), le sigue LMA M1 con el 17,5 \% (11) LMA M2 15,9\% (10), LMA M3 con el 12,7\%(8), LMA M4 con el 11,1\% (7), LMA M5 con el 9,5\%.
También se muestran los resultados de pacientes con mutación FLT3/ITD, la LMA M4 representa el 33,3\% (2) LMA M5 con un $33 \%$, LMA M0 y LMA M3 con el 16,7\% cada uno. (Ver Tabla 3).

Tabla 3. Subtipo morfológico según el diagnóstico de la mutación FLT3/ITD en pacientes atendidos en SOLCA de la ciudad de Cuenca periodo 2013-2019.

\begin{tabular}{|c|c|c|c|c|c|c|}
\hline \multirow{2}{*}{$\begin{array}{c}\text { SUBTIPO } \\
\text { MORFOLOGICO }\end{array}$} & \multicolumn{6}{|c|}{ MUTACIÓN FLT3/ITD } \\
\hline & $\mathbf{n}$ & $\%$ & $\mathbf{n}$ & $\%$ & $\mathbf{n}$ & $\%$ \\
\hline LMA MO & 13 & 20,6 & 12 & 21,1 & 1 & 16,7 \\
\hline LMA M1 & 11 & 17,5 & 11 & 19,3 & 0 & 0,0 \\
\hline LMA M2 & 10 & 15,9 & 10 & 17,5 & 0 & 0,0 \\
\hline LMA M4 & 7 & 11,1 & 5 & 8,8 & 2 & 33,3 \\
\hline LMA M3 & 8 & 12,7 & 7 & 12,3 & 1 & 16,7 \\
\hline LMA M5 & 6 & 9,5 & 4 & 7,0 & 2 & $33, \%$ \\
\hline LMA & 6 & 9,5 & 6 & 10,5 & 0 & 0,0 \\
\hline LMA M6 & 1 & 1,6 & 1 & 1,8 & 0 & 0,0 \\
\hline LMA M7 & 1 & 1,6 & 1 & 1,8 & 0 & 0,0 \\
\hline
\end{tabular}

En las características hematológicas de los pacientes con LMA se encontró una diferencia estadísticamente significativa en el porcentaje de blastos entre los pacientes positivos y negativos para FLT3/ITD, presentándose valores superiores en porcentaje de blastos en pacientes con la mutación con significación estadística. En lo que respecta a la cantidad de glóbulos blancos se presentan cifras anormales en los pacientes portadores de la mutación. (Ver Tabla 4). 
Tabla 4. Características hematológicas de los pacientes diagnosticados con LMA objetos de estudio, según el diagnóstico de la mutación FLT3/ITD atendidos en SOLCA de la ciudad de Cuenca periodo.

\begin{tabular}{|c|c|c|c|c|}
\hline \multirow{3}{*}{$\begin{array}{c}\text { VARIABLES } \\
\text { HEMATOLÓGICAS }\end{array}$} & \multirow[t]{2}{*}{ Todos } & \multicolumn{2}{|c|}{ Mutación ftl3 } & \multirow{3}{*}{ p. } \\
\hline & & Negativo & Positivo & \\
\hline & Mediana (RI) & Mediana (ri) & Mediana (ri) & \\
\hline $\begin{array}{l}\text { Glóbulos blancos } \\
\text { (CELS/MM3) }\end{array}$ & $8400(22150)$ & $8400(13315)$ & $41900(56150)$ & 0,140 \\
\hline $\begin{array}{l}\text { Hemoglobina } \\
\text { (MG/DL) }\end{array}$ & $8,60(2,20)$ & $8,50(1)$ & $10,05(3)$ & 0,140 \\
\hline $\begin{array}{l}\text { PLAQUETAS } \\
\text { (CELS/MM3) }\end{array}$ & $45000(96000)$ & $45000(44500)$ & $58500(63500)$ & 0,623 \\
\hline LDH (U/L) & 351 (578) & 314 (291) & 662 (547) & 0,121 \\
\hline \%BLASTOS & $61,54(58,64)$ & $59,72(29)$ & $89,43(5)$ & 0,031 \\
\hline
\end{tabular}

RI: Rango Interquartilico

En las variables clínicas, se apreció que un $58,7 \%$ de los individuos recayó y $38,1 \%$ falleció; con respecto a los individuos negativos para la mutación FLT3/ITD el 57,9\% recayeron y fallecieron el $35,1 \%$ y de los pacientes que portaban la mutación la mayoría recayó $(66,7 \%)$ y al igual que los individuos que fallecieron $(66,7 \%)$. (Ver Tabla 5).

Tabla 5. Variables clínicas de los individuos estudiados: supervivencia, remisión, muerte.

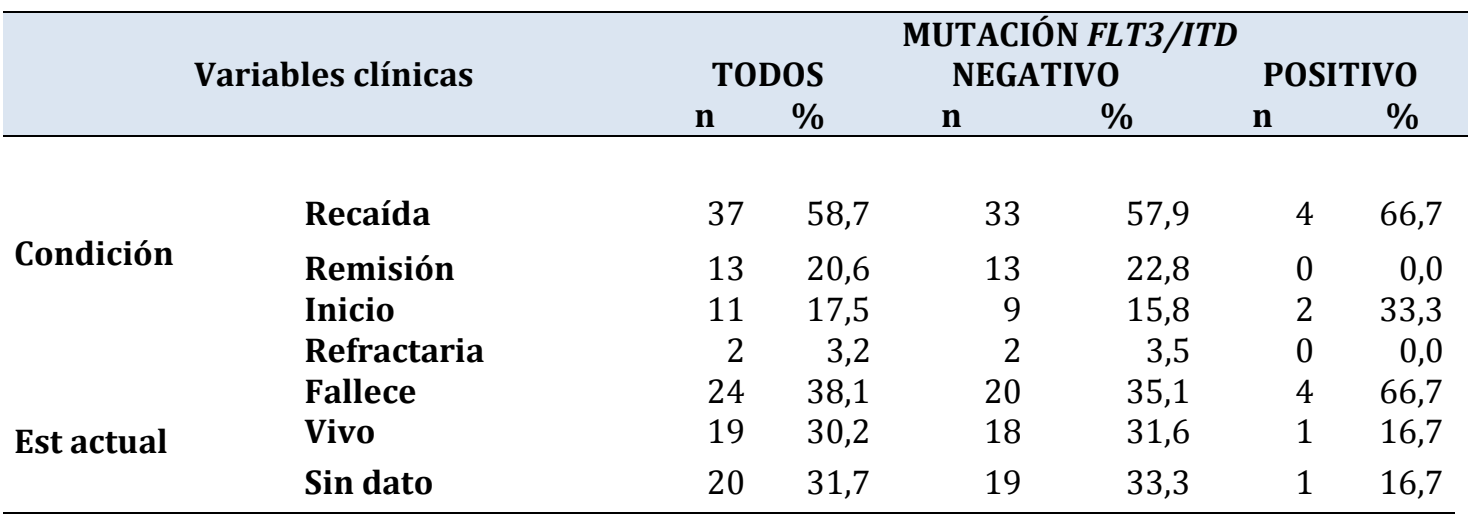

Se estudió la asociación entre las variables sexo, grupo etario, subtipo morfológico, glóbulos blancos, hemoglobina, plaquetas, LDH, remisión y muerte con la mutación FLT3/ITD encontrándose una asociación estadísticamente significativa en relación a la hemoglobina ya que los pacientes con FLT3/ITD tienen valores anormales de la misma demostrándose que los portadores de esta mutación tienen 6,63 más posibilidades de tener anormalidades en los niveles de hemoglobina (IC.1,00- 47,91, p. 0,037).También se encontró la LDH se encontraba elevada en 1,21 veces con mayor frecuencia en los portadores de la mutación, $(=p \quad 0,024)$ en pacientes positivos para la mutación con respecto a 
los negativos para mutación. En todas las demás variables evaluadas no se encontró asociación estadísticamente significativa con la mutación determinada en el presente estudio (Ver Tabla 6).

Tabla 6. Asociación de la presencia de la mutación FTL3-ITD con las características sociodemográficas, hematológicas y clínicas en pacientes objetos de estudio.

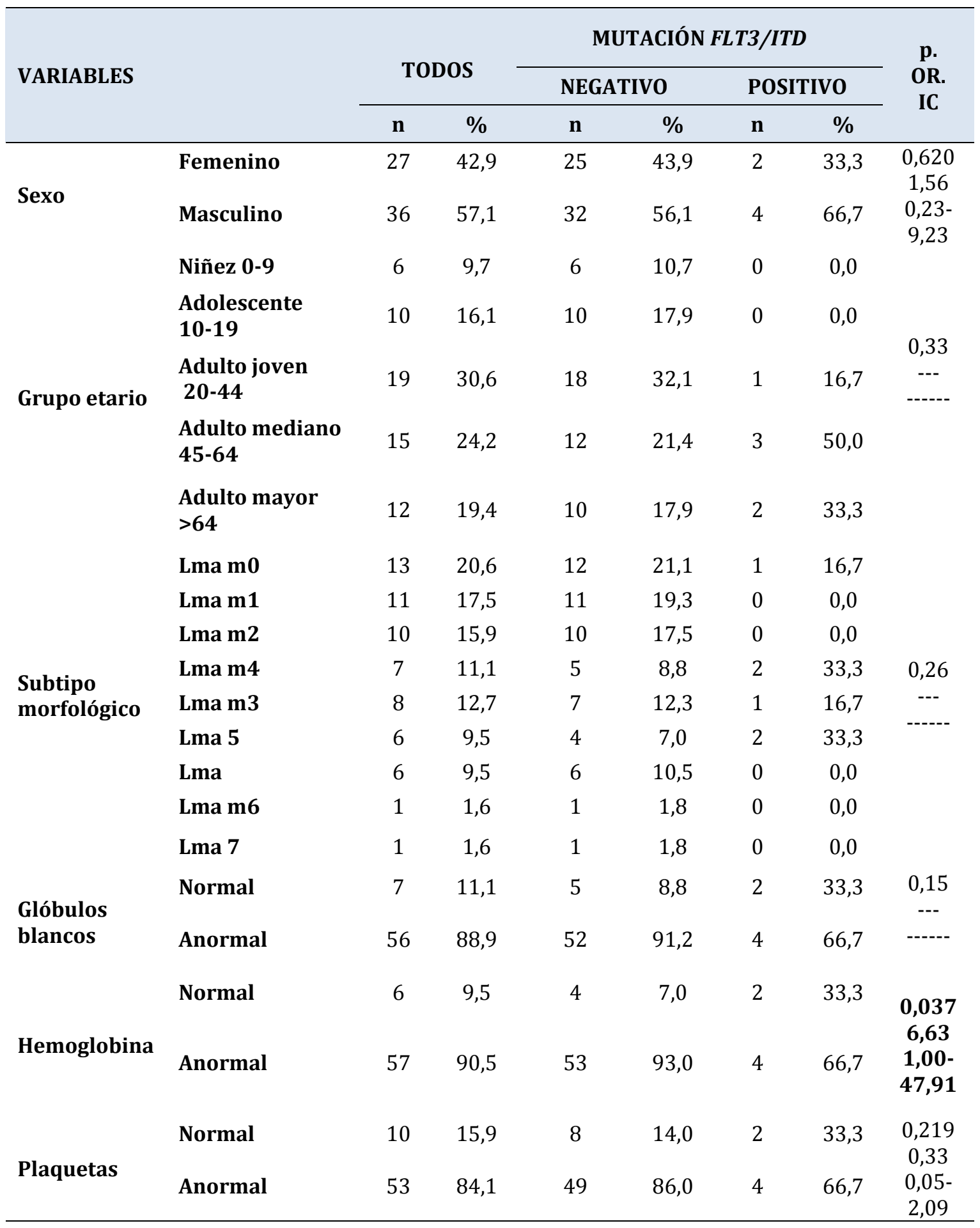




\begin{tabular}{|c|c|c|c|c|c|c|c|c|}
\hline \multirow{3}{*}{ VARIABLES } & & \multirow{2}{*}{\multicolumn{2}{|c|}{ TODOS }} & \multicolumn{4}{|c|}{ MUTACIÓN FLT3/ITD } & \multirow{3}{*}{$\begin{array}{c}\text { p. } \\
\text { OR. } \\
\text { IC }\end{array}$} \\
\hline & & & & \multicolumn{2}{|c|}{ NEGATIVO } & \multicolumn{2}{|c|}{ POSITIVO } & \\
\hline & & $\mathbf{n}$ & $\%$ & $\mathbf{n}$ & $\%$ & $\mathbf{n}$ & $\%$ & \\
\hline \multirow[b]{2}{*}{ Ldh } & Normal & 26 & 43,3 & 26 & 48,1 & 0 & 0,0 & $\begin{array}{c}0,024 \\
1,21\end{array}$ \\
\hline & Elevado & 34 & 56,7 & 28 & 51,9 & 6 & 100,0 & $\begin{array}{c}1,04- \\
1,42\end{array}$ \\
\hline \multirow{4}{*}{ Condición } & Recaída & 37 & 58,7 & 33 & 57,9 & 4 & 66,7 & \multirow{4}{*}{$\begin{array}{c}0,456 \\
--- \\
----\end{array}$} \\
\hline & Remisión & 13 & 20,6 & 13 & 22,8 & 0 & 0,0 & \\
\hline & Inicio & 11 & 17,5 & 9 & 15,8 & 2 & 33,3 & \\
\hline & Refractaria & 2 & 3,2 & 2 & 3,5 & 0 & 0,0 & \\
\hline \multirow{3}{*}{ Estado actual } & Fallece & 24 & 38,1 & 20 & 35,1 & 4 & 66,7 & \multirow{3}{*}{$\begin{array}{c}0,317 \\
----- \\
---\end{array}$} \\
\hline & Vivo & 19 & 30,2 & 18 & 31,6 & 1 & 16,7 & \\
\hline & Sin dato & 20 & 31,7 & 19 & 33,3 & 1 & 16,7 & \\
\hline
\end{tabular}

\section{DISCUSIÓN}

$\mathrm{L}$ as LMA representan entre 15 y el $20 \%$ de las leucemias agudas en niños y el $80 \%$ en adulto, la duplicación interna en tándem en el gen de la tirosina quinasa 3 FLT3/ITD, es una de las mutaciones de gran importancia para su estudio (1). Ya que esta alteración se relaciona con mayores tasas de recaída y disminución de supervivencia, con una prevalencia entre $20 \%$ y $27 \%$. FLT3/ITD es frecuente en pacientes con cariotipo normal y con anormalidad cromosómica de la t $(15,17)(4,7,8)$. Las LMA se asocian también con un alto porcentaje de células blásticas. Estudios de correlación detallan que la mutación FLT3/ITD se observa en pacientes masculinos preponderantemente con recuentos de leucocitos altos y LDH elevados, también se correlaciona en pacientes con FAB M1 y M4 $(11,12)$.
En el presente estudio se encontró en un total de 63 pacientes una frecuencia de 6 casos positivos que representa el 9.5\% para la mutación FLT3/ITD, en relación a la reportado a nivel mundial de FLT3-ITD en adultos con LMA que va desde el 15 al 35\% (14-16).

Jiménez et al (17). realizaron un estudio en 31 pacientes con LMA de novo en el cual reportan una prevalencia para FLT3-ITD de 9.4\%, estos datos concuerdan con este estudio ya que las muestras estudiadas son similares. En México RuizArguelles et al (18), encontraron una prevalencia de 13\%, Gaich et al (19). en Argentina señalan una incidencia del 16.7\%, Lucena et al (20). en Brasil, reportaron un 23.6\%. En Europa Gorin at el (21). informaron una prevalencia de $27.7 \%$ y Nazha et al (22). en Estados Unidos de 17.3\%. En Pakistan Ishsaq et al (5). encontraron la incidencia de ésta mutación en $4(13,3 \%)$ en LMA y en 1 (4\%) en ALL en un total de 55 pacientes. 
En 2015 Yanus et al (9) en un estudio con pacientes de LMA revelaron mutaciones dentro del dominio JM en el $13 \%(7 / 54)$, seis de ellos eran FLT3-ITD. Pajuela (11). Realizó la detección cualitativa de la mutación FLT3/ITD en un total de 389 pacientes, la cual fue detectada en 95 pacientes (24\%). Mahmood et al. (12). encontraron una frecuencia de la mutación FLT3-ITD del $18,5 \%$. Elyamany $G$ et al (23). han informado de una frecuencia del $14,4 \%$ en los pacientes con LMA de Arabia Saudita. Se encontraron mutaciones FLT3-ITD en el $19,2 \%$ de los pacientes con LMA en Tailandia por Kumsaen P et al. (12).

$\mathrm{Al}$ comparar estas investigaciones con los resultados que se obtuvieron en este estudio, destaca que la presencia de la mutación FLT3/ITD, es menor debido principalmente a la limitante de muestras con la que se trabajó en este estudio (63 pacientes). En un estudio realizado en Costa Rica entre 14 pacientes pediátricos con LMA encontraron una prevalencia del $14,3 \%$ para FLT3 mutado. En una población argentina pediátrica, Alonso et al (24). estudiaron a 92 pacientes con LMA y hallaron mutación FLT3 en $15,2 \%$ de pacientes, de estos, el 9,8\% tenía ITD. En este estudio, los niños con mutaciones FLT3 representaron solo el 7,1\% y todos tenían FLT3/ITD(10).en comparación con el presente estudio no se obtuvo resultados positivos de mutaciones en pacientes pediátricos, debido a que en la muestra de estudio, había 6 niños.

Así mismo al estudiar la presencia de la mutación FLT3/ITD y su relación con el sexo y edad, se encontró que el $66,7 \%$ de pacientes son de sexo masculino, prevalencia similar a la reportada en la literatura, además el grupo etario de mayor frecuencia fue el de adulto mediano
(45-64 años). Estudios realizados por Ishfaq et al. (5), indican que la mutación se presenta con mayor frecuencia en hombres que en mujeres y es más prevalente en pacientes de edad avanzada, este último dato difiere del estudio presente en donde el grupo etario más prevalente es el adulto mediano. Derek et al. informaron que la mutación FLT3 se detectó en el 27,5\% de los pacientes de 55 años, similar a lo informado por CuervoSierra, 29\% de la población de 60 años datos que son similares a este estudio (10).

Según las características hematológicas de los pacientes objetos de estudio, se encontró una diferencia estadísticamente significativa en el porcentaje de blastos, teniendo valores superiores los portadores de la mutación con una valor estadístico, (=p 0,031). Jiménez et al. (17). encontraron asociación entre el hallazgo de la mutación FLT3-ITD y el recuento elevado de leucocitos, en comparación con los pacientes libres de mutación $(\mathrm{p}=0.023)$. Esta investigación concuerda con la realizada por Burnatt et al (14) que indican que el recuento total de Glóbulos Blancos fue mayor para los pacientes que tenían la mutación FLT3-ITD y en el grupo pediátrico, también se observó una asociación significativa entre leucocitos elevados y la presencia de la mutación FLT3-ITD. Fröhling et al informaron de mayores recuentos de leucocitos y porcentajes de blastocitos en pacientes positivos para FLT3-ITD $(23,25)$. Por otro lado, Yanus et al. (9) informaron de una leucocitosis significativamente mayor en los pacientes con FLT3-ITD y en comparación con pacientes con FLT3 de tipo silvestre. Se ha indicado que la mutación FLT3-ITD suscita la activación constitutiva del receptor FLT3, lo que conduce a la estimulación celular 
independiente del ligando y la consiguiente proliferación incontrolada de blastos leucémicos (25).

Al analizar los subtipos morfológicos, se encontró que el subtipo más frecuente es LMA M0, además, este grupo fue negativo para la mutación FLT3/ITD.

En los casos con FLT3 mutados predominaron dos subtipos morfológicos LMA M4 con un 33,3\% y LMA M5 con un $33 \%$. Jiménez et al. (17) en un estudio realizado en 31 pacientes reportó que con respecto a la caracterización citogenética, dos de los tres pacientes con mutaciones FLT3-ITD tenían cariotipo normal (subtipos FAB M1,M2) y otro cariotipo complejo (subtipo FAB M5). En otra investigación, se indicó asociación de FLT3-ITD, con los subtipos FAB M1 y M4 $(\mathrm{p}=0.011)$ (11). Isfaq et al. (5) en su estudio informa que estas mutaciones se encuentran con mayor frecuencia en los subtipos M4 y M2 de LMA.

Este estudio también arrojó datos importantes referente a variables clínicas como la recaída y fallecimiento, con respecto a los individuos negativos para la mutación FLT3/ITD el 57,9\% recayeron y fallecieron el 35,1\% y los individuos que portaban la mutación la mayoría recayó $(66,7 \%)$ al igual que los individuos que fallecieron (66,7\%). En el año 2017 Burnatt et al (14). en un estudio realizado en Brasil, encontró asociación entre la presencia de la mutación FLT3-ITD y un mal resultado (muerte o recaída) en pacientes adultos. Sin embargo, el grupo pediátrico de LMA mostró una tendencia de asociación entre FLT3-ITD y muerte, lo que lo caracteriza como un indicador de mal pronóstico. en esta investigación no se encontró la mutación debido al bajo número de pacientes pediátricos.
Tao et al. (26) encontraron que la LMA con FLT3-ITD tiende a afectar a pacientes mayores con mayor frecuencia en el recuentos de glóbulos blancos, menor duración de la remisión y mayores tasas de recaída en comparación con los pacientes que no presentan la mutación.

Entre los hallazgos de este estudio, se encontró una asociación estadísticamente significativa en los valores de la hemoglobina, en pacientes con la mutación FLT3/ITD aumenta $(=\mathrm{p} 0,037)$ y un ratio 6,63 lo cual indica que el paciente tiene la posibilidad de tener 6,63 veces más elevada la hemoglobina con respecto a un paciente que no tienen la mutación. Trabajos similares indican que no encontraron correlación entre nivel de hemoglobina con FLT3 mutado en comparación con FLT3 silvestre (9) Zhang et al. (27) en su investigación concluye que no encontraron diferencias significativas en los recuentos de plaquetas $y$ hemoglobina en pacientes de los grupos estudiados, lo que sugiere que otras mutaciones simultánea y FLT3-ITD contribuyen a un aumento de glóbulos blancos y células progenitoras mieloides.

También se encontró la LDH elevada en 1,21 veces, $(=p 0,024)$ en pacientes que tienen la mutación con respecto a los que no tienen la mutación. En todas las demás variables evaluadas no se encontró asociación estadísticamente significativa con la mutación en el presente estudio. Al revisar la literatura, existen reportes en pacientes con Leucemias Aguda (LA), en los cuales se indica que un aumento de la actividad de la LDH se considera un factor de mal pronóstico (28). Un estudio con pacientes adultos de AL mostró una asociación entre la presencia de mutaciones del gen FLT3 y el aumento del 
nivel de LDH (29). Sin embargo, esta misma observación no se encontró en otro estudio (30). Burnatt (14) reporta que en el grupo de estudio (adultos y niños) los pacientes con mutaciones del gen FLT3 mostraron un aumento mayor de LDH. El incremento de la LDH está relacionada con lisis celular continua, así como, con proliferación anormal de clonas celulares siendo muy útil para confirmar invasión metastásica de neoplasias refractarias a tratamiento y como factor pronóstico adverso en LLA (31), aunque no se ha encontrado evidencia científica en individuos con LMA.

\section{CONCLUSIONES}

$\mathrm{E}$ $n$ este estudio se analizaron 63 pacientes con Leucemia Mieloide Aguda y se encontró que la presencia de la mutación FLT3/ITD fue de 9,5\% menor a la informada en la bibliografía. Además, se asoció un incremento en recuento de leucocitos, LDH y hemoglobina anormal en individuos portadores de la mutación.

En el presente estudio no se encontró una relación estadísticamente significativa con la clasificación FAB y otras variables clínicas: remisión, recaía y muerte. Esto puede deberse al reducido número de pacientes incluidos en el estudio y a las características de la población.

Para trabajos futuros es importante ampliar el número de pacientes de estudio, así como, apoyarse en métodos más sensibles como la secuenciación de nueva generación, que incrementaría las posibilidades de encontrar la relación entre éstas variables

\section{REFERENCIAS BIBLIOGRÁFICAS}

1. Muñoz D, Prada J, Castillo E. El Papel de FLT3 como Biomarcador en Leucemia Mieloide Aguda. Arch Med [Internet]. 2018;14(1). Disponible en: https://www.archivosdemedicina.com/ab stract/el-papel-de-flt3-como-biomarcadoren-leucemia-mieloide-aguda-22311.html

2. Lagunas F, Pérez V, Cortéz C. FLT3, NPM1 y C/EBP\&\#945; as prognosis markers in patients with acute myeloid leukemia. Rev Hematol. 2015;16(2):15267.

3. Amor A, Hernández L. La biología molecular en el diagnóstico de la leucemia mieloide aguda. Rev Cuba Hematol Inmunol Hemoter [Internet]. 2019;35(3). Disponible en: http://scielo.sld.cu/scielo.php?script=sci_a bstract\&pid=S0864$02892019000300002 \& \operatorname{lng}=\mathrm{es} \& \mathrm{nrm}=\mathrm{iso} \& \mathrm{t}$ lng=es

4. González E, Grille S, Vales V, Boada M, Zanella L, Leal D, et al. Estudio del ratio de FLT3-ITD como factor pronóstico en leucemias agudas mieloides: primeros casos estudiados en Uruguay. Rev Médica Urug. 2016;32(3):145-51.

5. Ishfaq M, Malik A, Faiz M, Sheikh I, Asif, Khan M, et al. Molecular Characterization of FLT3 Mutations in Acute Leukemia Patients. Asian Pac J Cancer Prev. 2012;13(9):4581-5.

6. Schnittger $S$, Bacher $U$, Haferlach $C$, Alpermann T, Kern W. Diversity of the juxtamembrane and TKD1 mutations (exons 13-15) in the FLT3 gene with regards to mutant load, sequence, length, localization, and correlation with biological data. Genes Chromosomes Cancer. 2012;51(10):910-24.

7. Lagunas F. Leucemia mieloide aguda. Una perspectiva de los mecanismos moleculares del cáncer. Gac Mex Oncol. 2016;15(3):150-7. 
8. Leyto F. Acute myeloid leukemia. Rev Hematol. 2018;19(1):24-40.

9. Yunus $\mathrm{N}$, Johan $\mathrm{M}, \mathrm{Al} \mathrm{H}$, Husin A, Hussein A, Hassan R. Characterisation and Clinical Significance of FLT3-ITD and non-ITD in Acute Myeloid Leukaemia Patients in Kelantan, Northeast Peninsular Malaysia. Asian Pac J Cancer Prev. 2015;16(12):4869-72.

10. Cuervo J, Jaime J, Martínez R, García R, Sánchez M, Gómez D, et al. Prevalence and Clinical Significance of FLT3 Mutation Status in Acute Myeloid Leukemia Patients: A Multicenter Study. Arch Med Res. 2016;47(3):172-9.

11. Gámez P, Carlos J. FLT3 y NPM1 como marcadores moleculares en la leucemia mieloblástica aguda. Utilidad pronóstica y diagnóstica. 2015; Disponible en: https://dialnet.unirioja.es/servlet/tesis?co digo $=75806$

12. Mahmood R, Altaf $C$, Malik $H$, Khan S. Clinico-Haematologic association and prognostic relevance of NPM1 and FLT3ITD mutations in acute Myeloid Leukaemia. Pak J Med Sci. 2019;35(1):238.

13. Cuervo J, Jaime J, Gómez D. FLT3 domain mutations in acute myelogenous leukemia. Rev Hematol. 2012;13(4):17784.

14. Burnatt $G$, Licínio $M$, Gaspar $P$, Ferreira A, Reis M, Moraes A, et al. Analysis of the presence of FLT3 gene mutation and association with prognostic factors in adult and pediatric acute leukemia patients. Braz J Pharm Sci [Internet]. 2017 [citado 12 de mayo de 2020];53(2). Disponible en: http://www.scielo.br/scielo.php?script=sc i_arttext\&pid=S1984-

82502017000200617

15. Gallogly $M$, Lazarus $H$, Cooper $B$. Midostaurin: a novel therapeutic agent for patients with FLT3-mutated acute myeloid leukemia and systemic mastocytosis. Ther Adv Hematol. 2017;8(9):245-61.
16. Abbas H, Alfayez M, Kadia T, RavandiKashani F, Daver N. Midostaurin In Acute Myeloid Leukemia: An Evidence-Based Review And Patient Selection. Cancer Manag Res. 2019;11:8817-28.

17. Jiménez A, Muskus C, Torres J, Cuéllar, Camargo, Vásquez G. Molecular characterization of FLT3-ITD mutations in Colombian patients with acute myelogenous leukemia. Rev Hematol. 2013;14(4):166-72.

18. Ruiz-Argüelles G, Garcés-Eisele, Alarcón- Urdaneta C, Lutz-Presno J, RuizDelgado G. Primary FMS-like tyrosine kinase 3 (FLT3) mutations in Mexican mestizo patients with de novo acute myelogenous leukemia. Presentado como cartel en XXXIV World Congress-ISH/LIII Congreso Nacional-AMEH, Cancún, abril de 2012. Citado en: Cuervo-Sierra J, JaimePérez JC, Gómez-Almaguer D. Mutaciones del módulo FLT3 en leucemia aguda mieloblástica. Rev Hematol Mex. 2012;13:177-84.

19. Gaich $\mathrm{P}$, Sastre $\mathrm{D}$, Rodríguez $\mathrm{C}$. Prevalencia de mutaciones flt3 en leucemias mieloblásticas agudas [Internet]. 2011. Disponible en: https://cobico.com.ar/wpcontent/archivos/PREVALENCIA-DEMUTACIONES-FLT3-ENLMA_Correccion.pdf

20. Lucena-Araujo A, Souza D, de Oliveira F, Benicio M, Figueiredo-Ponte Ls. Results of FLT3 mutation screening and correlations with immunophenotyping in 169 Brazilian patients with acute myeloid leukemia. Ann Hematol. 2010;89(2):225-8.

21. Gorin N, Labopin M, Meloni G, Esteve J, Mohamad M. Impact of FLT3 ITD/NPM1 mutation status in adult patients with acute myelocytic leukemia autografted in first remission. Haematologica. 2013;98(2):e12-4. 
22. Nazha A, Cortes J, Faderl S, Pierce S, Daver N. Activating internal tandem duplication mutations of the fms-like tyrosine kinase-3 (FLT3-ITD) at complete response and relapse in patients with acute myeloid leukemia. Haematologica. 2012;97(8):1242-5.

23. Elyamany G, Awad $M$, Fadalla $K$, Albalawi M, Al Shahrani M, Al Abdulaaly A. Frequency and Prognostic Relevance of FLT3 Mutations in Saudi Acute Myeloid Leukemia Patients. Adv Hematol. 2014;1-7.

24. Alonso C, Longo P, Medina A, Gallego $M$, Scopinaro M. Nuevos blancos terapéuticos en leucemia aguda pediatrica: caracterización de las mutaciones del gen FLT3. Med Infant. 2007;116-23.

25. Rezaei N, Arandi N, Valibeigi B, Haghpanah S, Khansalar M, Ramzi M. FMSLike Tyrosine Kinase 3 (FLT3) and Nucleophosmin 1 (NPM1) in Iranian Adult Acute Myeloid Leukemia Patients with Normal Karyotypes: Mutation Status and Clinical and Laboratory Characteristics. Turk J Hematol. 2017;34(4):300-6.

26. Tao S, Wang C, Chen $Y$, Deng $Y$, Song, Shi $Y$, et al. Prognosis and outcome of patients with acute myeloid leukemia based on FLT3-ITD mutation with or without additional abnormal cytogenetics. Oncol Lett. 2019;18(6):6766-74.

27. Zhang Q Z, Wu X, Caa J, Gao F, Huang K. Association between increased mutation rates in DNMT3A and FLT3-ITD and poor prognosis of patients with acute myeloid leukemia. Exp Ther Med. 2019;18(4):3117-24.

28. Varma N, Varma S. Proliferative indices, cytogenetics, immunophenotye and other prognostic parameters in myelodysplastic syndromes. Indian J Pathol Microbiol. 2008; 51(1):97.

29. Peng H, Zhang H, Gong F, Shen F, Zhang Y. Fms-like Tyrosine Kinase (FLT) 3 and FLT3 Internal Tandem Duplication in Different Types of Adult Leukemia: Analysis of 147 Patients. Croat Med J. 2008;49(5):650-9.

30. Karabacak B, Erbey F, Bayram I, Yilmaz S, Acipayam C. Fms-like tyrosine kinase 3 mutations in childhood acute leukemias and their association with prognosis. Asian Pac J Cancer Prev. 2010; 11(4):923-7.

31. Moya $S$, Pio D. Parámetros bioquímicos enzimáticos (ALT, AST, ALP, $\gamma$-GT, LDH) en niños con leucemia linfoblástica aguda antes del tratamiento antineoplásico. Horiz Méd Lima. 2015; 15(4):52-8.

Conflicto de intereses. Los autores declaran que no existe conflicto de intereses para la publicación del presente artículo.

Financiamiento. Autofinanciamiento

Agradecimiento. No declaran 